\title{
THE \\ PSYCHOLOGICAL BULLETIN
}

\section{GENERAL}

714. ZIEHEN, T., Die Beziehungen der Lebenserscheinungen zum Bewusstsein. Abhandl. z. theoretischen Biol., Heft 13 Berlin, $1921,66$.

Verf. erörtert unter kritischer Berïcksichtigung des Vitalismus den Begriff der Lebenserscheinungen und hierauf den Begriff des Bewusstseins. Insbesondere wird die Frage unbewusster psychischer Prozesse ausführlich behandelt (Einteilung der bewussten Prozesse in attendierte und nicht-attendierte, der attendierten in egotale und anegotale, d.h. mit oder ohne Denken einer Ich-Beziehung; unbewusste psychische Prozesse werden abgelehnt). Es folgt dann die Besprechung der Kriterien für das Vorhandensein von Bewusstseinsprozessen und eine eingehende Erörterung, wie weit die Zuordnung von Bewusstseinsprozessen sich innerhalb des menschlichen Körpers, innerhalb der Tierreihe erstreckt usf. Hier sei nur erwähnt, dass Verf. die Beschränkung der Bewusstseinserscheinungen auf die Grosshirnrinde im üblichen Sinn bekämpft und in den erkenntnistheoretischen Schlussbemerkungen den hylopsychismus vertritt.

TH. ZienEN (Halle)

715. Friedmann, C., Psychologische Momente in der Ableitung des Apriori bei Kant. Kant-Studien, 1921, 26, 312-350.

Die Marburger Schule und auch Windelband nehmen ein in unserer Vernunft begründetes Apriori als Voraussetzung unserer wissenschaftlichen Erfahrung an. Diese Richtung lehnt das Entwicklungsprinzip zur Aufdeckung des Apriori ab. Dagegen wollen die Anhänger der kritischpsychologischen Philosophie das Entstehen des transzendentalen Apriori. aufzeigen. Sie analysieren nach psychogenetischer Methode die in der Erfahrung gewonnene Gegebenheit. Sie berufen sich auf das in der Erfahrung gewordene Apriori. Es wird gezeigt, dass Kant selbst sowohl die von ihm geschaffene trans- 
zendentale als auch die psychologische Methode verwendet. In der Ableitung der reinen Anschauungsform des Raumes wird eine Verwechslung von Abstraktion mit Vorstellungsmöglichkeit gesehen. In der Nichtvorstellbarkeit des Raumes und der Nichthinwegdenbarkeit der Zeit soll das psychologische Motiv zu suchen sein. Auch die transzendentale Apperzeption schöpft aus dem Erlebnis Ebenso beruht die Notwendigkeit des Kausalitätsgesetzes auf einem psychologischen Motiv und stammt aus dem Erlebnis des Handelns und Wirkens. Bei Kant verschmilzt die psychologische Notwendigkeit mit der logischen transzendentalen Notwendigkeit und dies wird als Versöhnungsmotiv der verschiedenen Richtungen der Kantschen Philosophie angesehen.

\section{HABRICHT (München)}

716. Wrchmans, O., Genie und Tragik. Kantstudien, 1921, 26, 351-389.

Das Wesen der Tragik wird gesucht in einem eigentümlichen Verhältnis von Freiheit und Notwendigkeit: Freiheit von den Bedingungen des gewöhnlichen Lebens, sich steigernd zur Unbedingtheit des wirklich unbedingten-dämonischen (Plato, Göethe) - Wesens, oder im Willen zur Freiheit die eigene Begrenztheit zur Unbedingtheit erhebend. Notwendigkeit-Unabwendbarkeit des Unterganges-prägt sich also umso mehr in einem Schicksal aus, je stärker der Drang nach Freiheit, nach Unbedingtheit ist. Die Behandlung der Darstellungen in Theorie, Werk und Leben grosser Dichter und Denker rechtfertigt den Titel ebensosehr, wie die feinsinnigen Ausführungen über, "Freiheit als Unbedingtheit." Die, Mythisierung dieses Jenseitig-Unbedingten in dèr menschlichen Natur in Form der Vergöttlichung (Antike Tragödie), wie als übersinnliches in der Seele des tragischen Menschen sind die weiteren Themen dieser inhaltsreichen Untersuchung.

\section{B. KLOPFER (München)}

717. Stern, W., Der Formvariator. Zeits. f. pädag. Psychol., 1922, 23, 131-137.

Beschreibt ein vom Verfasser konstruiertes Hilfsmittel zur Prüfung und Erziehung der dynamisch geometrischen Raumauffassung, das im psychologischen Laboratorium der Hamburgischen Universität verwendet wird. Der "Variatorwürfel " ist ein Gerüst aus 12 Metallstäben mit elastischen Eckenverbindungen. $\mathrm{Er}$ 
gestattet die mannigfachsten Umwandlungen in zwei- und dreidimensionale Formen. Er ermöglicht eine Diagnostizierung des für gewisse Berufsarten wesentlichen der Sonderbegabung, sich nicht nur ruhende Formen, sondern auch ihre Verschiebungen und Projektionen, ihre durch bestimmt gerichtete Kräfte bewirkte Uberleitung in andere Formen innerlich vorzustellen.

\section{H. Bogen (Berlin)}

LIPPMANN, O., Bibliographie zur psychologischen Berufsberatung, Berufseignungsforschung und Berufskunde. Unter Mitwirkung von Franziska Baumgarten. Schriften z. Psychol. d. Berufseignung u. d. Wirtschaftslebens. Heft 20, 1922. $60 \mathrm{~S}$.

Die Bibliographie enthält, alphabetisch nach Verfassernamen geordnet, etwa 900 Titel von teils wissenschaftlichen, teils populären Arbeiten, Zeitungsartikeln usw. der deutschen und ausländischen Literatur, nebst einem Hinweis auf wichtige Referate. Durch ein sehr eingehendes "Sachverzeichnis," in dem auf die angeführten Arbeiten verwiesen wird, wird versucht. ein System des behandelten Wissengebietes zu schaffen. Die Benutzung der Bibliographie wird auch dadurch erleichtert, dass die mehrfach vorkommenden Zeitschriften-Titel zusammengestellt sind, und dass dabei auf alle in der betr. Zeitschrift enthaltenen citierten Arbeiten verwiesen wird. Die Bibliographie wird im Institut für angewandte Psychologie fortgeführt.

$$
\text { O. LipMANN (Berlin) }
$$

719. BAAdE, W., Eine Korrelationsrechenmaschine. Zeits. f. angewe. Psychol., 1922, 20, 223-225.

Die Vorrichtung gestattet ein sehr schnelles Errechnen des Korrelationskoeffizienten nach der Spearmanschen Formel. Auf die Schale einer Zeigerwage sind Gewichte aufzulegen, deren Schwere proportional ist dem Quadrat der Rangplatzdifferenzen. Der Koeffizient kann dann an der Skala abgelesen werden.

H. Bogen (Berlin)

720. Baumgarten, F., Die “II. Internationale Konferenz für Psychotechnik, angewandt auf Fragen der Berufsberatung und Arbeitsorganisation" in Barcelona. (28-30 September, 1921.) Zeits. f. angew. Psychol., 1922, 20, 248-258.

H. Bogen (Berlin) 
721. Plaut, P., Zur Psychologie des Krieges. Bibliographischer Sammelbericht. Zeits. f. angew. Psychol., 1922, 20, 281286.

Enthält ausführlichen Nachtrag zur Bibliographie des Verfassers in: Beiheft z. Zeits. f. angew. Psychol., Nr. 21, 1920.

H. BoGEN (Berlin)

722. Stern, W., Das psychologische Laboratorium der Hamburgischen Universität. Gesamtbericht über seine Entwicklung und seine gegenwärtigen Arbeitsgebiete. Zeits. $f$. pädag. Psychol., 1922, 23, 161-196. 1922.

Als Sonderabdruck erschienen bei Quelle \& Meyer, Leipzig,

H. Bogen (Berlin)

723. Hunziker, H., Vom Wert der Zensuren. Schweizer. pädag. Zeits., 1922, 32, 39-45.

Verf. hat Zensurenstatistiken nach Lipps'schen psychophysischen Massmethden verarbeitet, um den Wert der Zensur als Leistungsgradmesser zu überprüfen. Es ergibt sich eine recht geringe Schwankung der mittleren Werte erster Ordnung beim Vergleich der mittleren Klassennoten eines Lehrers in mehreren aufeinanderfolgenden Jahren. Der "strengste" Lehrer beurteilt im Durchschnitt nur um eine halbe Zensurstufe neidriger als der "gefälligste" Lehrer. Dieses Ergebnis trifft dann nicht ganz zu, wenn man die Differenzen zwischen zwei Klassen bei Beurteilung durch denselben Begutachter vergleicht mit denen gleicher Altersstufen durch verschiedenen Begutachter; sie sind dann im ersteren Falle leicht grösser als im letzteren. Mit zunehmendem Alter nähert sich die Verteilung der schlechten und guten Fälle immer mehr dem Wert an, der die symmetrische Verteilung darstellt. "Man darf sagen, dass die Lehrer ihre Schüler in hohem Grade ähnlich, um ticht geradezu zu sagen gleichartig zensieren."

H. BogEN (Berlin)

724. OTIS, A. S., A Method of Inferring the Change in a Coefficient of Correlation Resulting from a Change in the Heterogeneity of the Group. J. of Educ. Psychol., 1922, 13, 293-294.

Otis presents a formula for determining the coefficient of correlation to be expected from a given group when the correlation for a 
group of different heterogeneity is known. This formula is derived from the Otis Difference formula previously published.

A. T. Poffenberger (Columbia)

725. Ruckmick, C. A., A Cabinet for Colored Papers. Science, 1922, 56, 76-77.

$$
\text { G. J. RICH (Pittsburgh) }
$$

726. Ginzburg, B., The Quest for Objectivity in the Study of Human Phenomena. J. of Abnor. Psychol. and Social Psychol., 1922, 16, 367-373.

The article begins by pointing out the incompleteness and inconsistency of the behavioristic system, quoting the views of several French authorities on the value of introspective psychology. It then passes to the discussion of the work of a group of French thinkers who "have shown the fallacy of studying the individual isolated from his social frame-work," and the institution of the "Sociological School." An account is given of its brilliant but brief existence.

D. A. Macfartane (Boston Psychopathic Hospital)

\section{SENSATION AND PERCEPTION}

727. Noltenius, F., Raumbild und Fallgefühl im Fluge. Arch. $f$. Ohren-Nasen und Kehlkopfheilk., 1922, 108, 107-126.

Der Verf. berichtet über Beobachtungen aus seiner Fliegerzeit und Versucht sie in die herrschenden Theorien über das Zustandekommen des Raumbildes und der Orientierung des eigenen Körpers einzufügen. Zwar geht er mit Vorliebe auf die langst überlebte Urteilstheorie zurück, dergemäss bei Sinnestäuschungen das Urteil gewaltsam in die Kuppelung der Lokalisationskomplexe eingreifen sollen. Dafür bringenaber die Einzelbeobachtungen selbst mancherlei veues. Insbesondere tritt die Fallempfindung nur bei senkrechter Kopfhaltung auf, sie fehlt wenn die Kopfasche horizontal steht. Dies soll sich aus der besonderen Tätigkeit des Statolithenorgans erklären das beim Aufrechtstehen dauernd den Tonus der Muskulatur erhält. Beim senkrechten Fall wird die Spannung der Sinneshaare in dem Statolithenorgan aufge entspannt und damit jener Tonus der Muskulatur aufgehoben Beim Fall mit horizontaler Körperachse 